\title{
Tensor and matrix models: a one-night stand or a lifetime romance?
}

\author{
Pablo Diaz \\ Fields, Gravity 8 Strings, CTPU, Institute for Basic Science, \\ Daejeon 34126 KOREA \\ E-mail: pablodiazbe@gmail.com
}

ABSTRACT: The spectra of energy eigenstates of free tensor and matrix models are organized by Kronecker coefficients and Littlewood-Richardson numbers, respectively. Exploiting recent results in combinatorics for Kronecker coefficients, we derive a formula that relates Kronecker coefficients with a hook shape with Littlewood-Richardson numbers. This formula has a natural translation into physics: the eigenstates of the hook sector of tensor models are in one-to-one correspondence with fluctuations of $1 / 2$-BPS states in multi-matrix models. We then conjecture the duality between both sectors. Finally, we study the Hagedorn behaviour of tensor models with finite rank of the symmetry group and, using similar arguments, suggest that the second (high energy) phase could be entirely described by multi-matrix models.

Keywords: Duality in Gauge Field Theories, Matrix Models, Gauge-gravity correspondence

ArXiv EPrint: 1803.04471 


\section{Contents}

1 Introduction $\quad 1$

$\begin{array}{llr}2 & \text { Tensor partition function } & 2\end{array}$

3 Kronecker coefficients with a hook shape and multi-matrix models $\quad 6$

4 Hagedorn phase transition for finite $N \quad 10$

5 Summary and outlook $\quad 12$

A Kronecker coefficients with a hook shape: corner approximation $\quad 13$

\section{Introduction}

There are several motivations for the study of tensor models in theoretical physics. From the Quantum Mechanics point of view, tensor models are expected to suit a description of entangled systems [1,2]. From the quantum gravity perspective, and inspired by the success of matrix models in the description of two-dimensional quantum gravity [3], tensor models were proposed in the early 90's as a framework for studying higher dimensional quantum gravity [4-6]. Recently, the interest in tensor models has been boosted in the context of $\mathrm{AdS}_{2} / \mathrm{CFT}_{1}$ were the SYK model [7-17] has been shown to share the same large $N$ behaviour as a tensor model [18]. Besides, the arrival of color tensor models [19, 20], rainbow models [21, 22] and multi-orientable models [23], together with the understanding of their 1/ $N$ expansion [24-29] (which helped to resolve old large $N$ issues) has certainly triggered the rapid development of the subject in the last years.

In this work we plan to contribute to the development of tensor models and the physics they involve by putting them in contact with matrix models. In this regard, there are at least two hints which make us suspect that a connection (probably deep) between tensor and matrix models exists:

1. The holographic conjecture of the SYK model in the tensor model version [18] and the increasing suspicion that tensor models have holographic duals in broader contexts, seem to indicate that there should be an overlap between tensor and matrix models, since the latter have been proven to encode holographic duals at least in the large $N$ regime. Actually, finding the precise relation between both models will be extremely interesting for holography. On the one hand the dictionary between matrix models and gravity duals is well-understood nowadays but the perturbative expansion for multi-matrix models is highly complicated. On the other hand, tensor models with quartic interaction present an easy-to-tackle (melonic) perturbative 
expansion but its relation with gravity is still unknown. Therefore, understanding the connection between both theories will bring insights into holography and perhaps new computational tools.

2. The recent discovery of the relation between some sectors of Kronecker coefficients and Littlewood-Richardson numbers in the field of combinatorics and group theory. This is relevant for us since Kronecker coefficients organize the spectrum of eigenstates of free tensor models [30-33], whereas Littlewood-Richardson numbers have long been known to organize the spectrum of matrix models [35-42].

Based on (partially) matching the spectrum of the free theory in both models through the above-mentioned relation between Kronecker coefficients and Littlewood-Richardson numbers we conjecture in this paper that the corresponding tensor and matrix sectors are dual. Note that matching the spectrum is a necessary condition for duality, for a definite proof we should see that those sectors are dynamically equivalent, a programme that we leave for a future work.

The paper is organized as follows. We start by writing and evaluating the partition function with the singlet condition of the free tensor model in section 2. The spectrum of energy eigenstates is organized by Kronecker coefficients, as can be read from eq. (2.22). This is in perfect agreement with the direct counting of invariants found in recent works. In section 3, we gather some results on combinatorics of Kronecker coefficients and we derive (3.3), which tells the explicit relation between the hook sector of Kronecker coefficients and Littlewood-Richardson numbers. We then manage to construct the matrix operators that those numbers count and we arrive at (3.11), which is the main result of the paper. Eq. (3.11) tells us that the hook sector of the tensor model has the same number of eigenstates than a rather general multi-matrix set of operators. These multi-matrix operators are interpreted as encoding fluctuations about a generic $\frac{1}{2}$-BPS state of a depth given by the length of the hook in the tensor sector. In short, the hook sector of the tensor spectrum

encodes the fluctuations of $\frac{1}{2}$-BPS states in the matrix theory. In section 4 , we return to the partition function. Tensor models with finite rank of the symmetry group are known to have Hagedorn behaviour, a fact that is interpreted as a phase transition at some finite temperature related to the rank of the group. We study the growth of states of the second (high energy) phase of tensor models and, using a known theorem of Kronecker coefficients, we conjecture that this second phase can be entirely described by a multi-matrix model. Finally, we include an appendix for the computation of Kronecker coefficients with a hook shape in the regime of large (but finite) rank.

\section{Tensor partition function}

Color tensors refer to tensors with no additional symmetry assumed. We will write a $d$-rank covariant color tensor as

$$
\Phi=\Phi_{i_{1} i_{2} \ldots i_{d}} e^{i_{1}} \otimes \cdots \otimes e^{i_{d}}
$$


where the complex-valued vectors $\left\{e^{i_{k}}, i_{k}=1, \ldots, N_{k}\right\}$ form an orthonormal basis of the vector space $\mathbb{C}^{N_{k}}$. The components of the tensor transform under the action of

$$
G_{d} \equiv \mathrm{U}\left(N_{1}\right) \otimes \cdots \otimes \mathrm{U}\left(N_{d}\right)
$$

as

$$
\Phi_{j_{1} j_{2} \ldots j_{d}}=\sum_{i_{1}, \ldots, i_{d}} \mathrm{U}\left(N_{1}\right)_{j_{1}}^{i_{1}} \cdots \mathrm{U}\left(N_{d}\right)_{j_{d}}^{i_{d}} \Phi_{i_{1} i_{2} \ldots i_{d}}
$$

The complex conjugate of $\Phi$ is a $d$-rank contravariant tensor which transforms as

$$
\bar{\Phi}^{j_{1} j_{2} \ldots j_{d}}=\sum_{i_{1}, \ldots, i_{d}} \overline{\mathrm{U}}\left(N_{1}\right)_{i_{1}}^{j_{1}} \cdots \overline{\mathrm{U}}\left(N_{d}\right)_{i_{d}}^{j_{d}} \bar{\Phi}^{i_{1} i_{2} \ldots i_{d}}
$$

The action of the free theory is simply

$$
S=\Phi_{i_{1} i_{2} \ldots i_{d}} \bar{\Phi}^{i_{1} i_{2} \ldots i_{d}}
$$

Invariant operators are $n$-fold tensor products $\Phi^{\otimes n} \bar{\Phi}^{\otimes n}$, built out of $n$ copies of the tensor $\Phi$ contracted with $n$ copies of its conjugate. Each invariant is associated with the specific way indices of $\Phi$ and indices of $\bar{\Phi}$ are contracted subjected to a double coset equivalence, see [43] for details. Counting the number of invariant operators, building a basis which diagonalizes the two-point function of the free theory and computing correlators has been a recent subject of study [30-34]. In those studies it was manifest the prominent role of Kronecker coefficients in organizing the spectrum of energy eigenstates.

The aim of this section is to compute the number of invariants by evaluating the partition function. The first question one can ask is if there is any sense of a partition function at all in a theory where there is no time, like the tensor model corresponding to the action (2.5). In fact, one usually defines

$$
Z(\beta)=\operatorname{Tr}\left(e^{-\beta H}\right)
$$

Even if we prefer, as common, to use the equivalent Euclideanized action to define the partition function, it is still the time coordinate which must be Wick rotated and compactified. Thus, we need to consider our fields to depend on time. The dynamics are given by a suitable action that we define below.

The crucial point is that, although the theories (with time and no time) are different, they count the same number of $G_{d}$-invariants, since the number of invariants count the ways of contracting the internal indices. For instance, with two fields there is only one $G_{d^{-}}$-invariant possible at a given time, which is $\Phi_{i_{1} i_{2} \ldots i_{d}}(t) \bar{\Phi}^{i_{1} i_{2} \ldots i_{d}}(t)$, and similarly for the $n$-fold composites.

So, in order to count invariants we will use the free tensor theory given by the action

$$
S=\int d t \frac{d \Phi_{i_{1} i_{2} \ldots i_{d}}}{d t} \frac{d \bar{\Phi}^{i_{1} i_{2} \ldots i_{d}}}{d t}-\omega^{2} \Phi_{i_{1} i_{2} \ldots i_{d}}(t) \bar{\Phi}^{i_{1} i_{2} \ldots i_{d}}(t)
$$


The Hamiltonian of this theory is a collection of harmonic oscillators (actually two decoupled collections, since the field is complex), labeled by $G_{d}$, which can be written schematically as

$$
H=\sum_{\alpha} \omega\left(a_{\alpha}^{\dagger} a_{\alpha}+b_{\alpha}^{\dagger} b_{\alpha}\right)
$$

where $\alpha$ is a label for the group.

The partition function whose evaluation will give us the number of invariants must implement the singlet condition, otherwise it will account for all the states of the theory, not only $G_{d}$-invariants. For that we will need to average over $G_{d}$. So, the partition function we will consider is

$$
Z_{s}(\beta)=\sum_{i} \int_{G_{d}} \mathrm{~d} g_{1} \mathrm{~d} g_{2}\left\langle\Gamma\left(g_{1}\right) i\left|e^{-\beta H}\right| \Gamma\left(g_{2}\right) i\right\rangle,
$$

where $i$ runs over a basis of states, and the Hamiltonian is (2.8). The subscript $s$ in $Z_{s}$ stands for "singlet". The representation $\Gamma(g)$ of $G_{d}$ acts on the states and will be specified below. $\mathrm{d} g$ is the Haar measure.

Equation (2.9) was first proposed in [44], where it was shown that it leads to

$$
Z_{s}(\tau)=\int_{G_{d}} \mathrm{~d} g \exp \left(\sum_{n=1}^{\infty} \frac{1}{n} \tau^{n} \chi\left(g^{n}\right)\right), \quad \tau=e^{-\beta \omega},
$$

where $\chi(g)$ is the character of the representation $\Gamma(g)$ of $G_{d}$.

Although we have been calling it $G_{d}$, the partition function (2.10) is actually general, valid for any symmetry group $G$ and for any representation of the fields. In our case, the group is $G_{d}=\mathrm{U}\left(N_{1}\right) \times \cdots \times \mathrm{U}\left(N_{d}\right)$. As said before, the gauge invariant operators transform under the fundamental-antifundamental representation of $G_{d}$, so

$$
\chi(g)=\operatorname{tr}(g) \operatorname{tr}\left(g^{+}\right), \quad g \in G_{d} .
$$

Now, since $\chi(g)$ is independent of the choice of basis, we will choose, for every $\mathrm{U}\left(N_{k}\right)$, a basis where the matrix is diagonal. We will make use the of the Weyl parametrization of $\mathrm{U}\left(N_{k}\right)$ and use $u_{k} \in \mathrm{U}\left(N_{k}\right)$ to be $u_{k}=\operatorname{diag} .\left(e^{i \theta_{k 1}}, \ldots, e^{i \theta_{k N_{k}}}\right)$, with $0 \leq \theta_{k i} \leq 2 \pi$. With this parametrization we will write a group integral as

$$
\int_{G_{d}} \mathrm{~d} g F(g)=\prod_{k=1}^{d} \frac{1}{(2 \pi)^{N_{k} N_{k} !}} \int_{0}^{2 \pi} \prod_{j=1}^{N_{k}} \mathrm{~d} \theta_{k j} \prod_{1 \leq l, m \leq N_{k}}\left|e^{i \theta_{k l}}-e^{i \theta_{k m}}\right|^{2} F\left(u_{k}\right) .
$$

We will use the following convenient notation for the eigenvalues $z_{k j}=e^{i \theta_{k j}}$ and also use the string of eigenvalues

$$
z_{k} \equiv\left(z_{k 1}, \ldots, z_{k N_{k}}\right)
$$

First, let us notice that with this parametrization and using general properties of the Kronecker product of matrices, the character (2.11) of a general element $g \in G_{d}$ can be written in terms of symmetric functions of the eigenvalues as

$$
\chi\left(g^{n}\right)=\operatorname{tr}\left(g^{n}\right) \operatorname{tr}\left(\left(g^{+}\right)^{n}\right)=p_{n}\left(z_{1} z_{2} \cdots z_{d}\right) p_{n}\left(z_{1}^{-1} z_{2}^{-1} \cdots z_{d}^{-1}\right)
$$


where $p_{n}$ are the power sums and the string $z_{1} z_{2} \cdots z_{d}$ stands for a collection of $\prod_{k=1}^{d} N_{k}$ variables of the type $z_{1 i_{1}} \cdots z_{d i_{d}}$ with $1 \leq i_{k} \leq N_{k}$. We will also use the notation

$$
\Delta\left(z_{k}\right)=\prod_{1 \leq i, j \leq N_{k}}\left(z_{k i}-z_{k j}\right)
$$

for the Vandermonde determinant. Thus, the partition function of the free tensor model can be written as an integral over complex eigenvalues as

$Z_{s}(\tau)=\prod_{k=1}^{d} \frac{1}{(2 \pi)^{N_{k} N_{k} !}} \oint \prod_{i=1}^{N_{k}} \frac{\mathrm{d} z_{k i}}{z_{k i}} \Delta\left(z_{k}\right) \Delta\left(z_{k}^{-1}\right) \exp \left(\sum_{n=1}^{\infty} \frac{\tau^{n}}{n} p_{n}\left(z_{1} \cdots z_{d}\right) p_{n}\left(z_{1}^{-1} \cdots z_{d}^{-1}\right)\right)$.

By Taylor expansion and reordering terms, it is not hard to see that the exponential in (2.16) can be expressed as a sum over partitions, that is,

$$
\exp \left(\sum_{n=1}^{\infty} \frac{\tau^{n}}{n} p_{n}\left(z_{1} \cdots z_{d}\right) p_{n}\left(z_{1}^{-1} \cdots z_{d}^{-1}\right)\right)=\sum_{n=1}^{\infty} \sum_{\lambda \vdash n} \frac{1}{z_{\lambda}} \tau^{n} p_{\lambda}\left(z_{1} \cdots z_{d}\right) p_{\lambda}\left(z_{1}^{-1} \cdots z_{d}^{-1}\right) .
$$

Using the relation between power sums and Schur functions

$$
p_{\lambda}(z)=\sum_{\mu \vdash n} \chi_{\mu}(\lambda) s_{\mu}(z)
$$

we may write

$$
\begin{aligned}
& \exp \left(\sum_{n=1}^{\infty} \frac{\tau^{n}}{n} p_{n}\left(z_{1} \cdots z_{d}\right) p_{n}\left(z_{1}^{-1} \cdots z_{d}^{-1}\right)\right) \\
= & \sum_{n=1}^{\infty} \sum_{\lambda, \mu, \nu \vdash n} \frac{1}{z_{\lambda}} \tau^{n} \chi_{\mu}(\lambda) \chi_{\nu}(\lambda) s_{\mu}\left(z_{1} \cdots z_{d}\right) s_{\nu}\left(z_{1}^{-1} \cdots z_{d}^{-1}\right) .
\end{aligned}
$$

Schur functions of the variables $z_{1} \cdots z_{d}$ (remember that they are $N$ variables) can be written as [45]

$$
s_{\mu}\left(z_{1} \cdots z_{d}\right)=\sum_{\mu_{1}, \ldots, \mu_{d} \vdash|\mu|} g_{\mu, \mu_{1}, \ldots, \mu_{d}} s_{\mu_{1}}\left(z_{1}\right) \cdots s_{\mu_{d}}\left(z_{d}\right) .
$$

The point of writing the exponential, and so the partition function in terms of Schur functions this way is because we can apply straightforwardly the explicit inner product of Schur functions [46]

$$
\delta_{\mu \nu}=\left\langle s_{\mu}, s_{\nu}\right\rangle_{N_{k}}=\frac{1}{(2 \pi i)^{N_{k}} N_{k} !} \oint \prod_{i=1}^{N_{k}} \frac{\mathrm{d} z_{k i}}{z_{k i}} \Delta\left(z_{k}\right) \Delta\left(z_{k}^{-1}\right) s_{\mu}\left(z_{k}\right) s_{\nu}\left(z_{k}\right), \quad l(\mu), l(\nu) \leq N_{k} .
$$

It is important to stress that Schur functions $s_{\mu}(z)$ are identically 0 whenever $l(\mu)$ is greater than the number of variables, as indicated in (2.21). This will restrict the sums over partitions in the following. By making first the substitution of the exponential (2.19) 
with (2.20) in the partition function (2.16), and then applying (2.21) to each pair of Schur functions we arrive at

$$
\begin{aligned}
& Z_{s}(\tau)=\sum_{n=1}^{\infty} \sum_{\substack{\lambda, \mu, \nu \vdash n \\
\mu_{1}, \ldots \mu_{d} \vdash n \\
\nu_{1}, \ldots \nu_{d} \vdash n \\
l\left(\mu_{k}\right), l\left(\nu_{k}\right) \leq N_{k}}} \frac{1}{z_{\lambda}} \tau^{n} \chi_{\mu}(\lambda) \chi_{\nu}(\lambda) g_{\mu, \mu_{1}, \ldots, \mu_{d}} g_{\nu, \nu_{1}, \ldots, \nu_{d}} \delta_{\mu_{1} \nu_{1}} \cdots \delta_{\mu_{d} \nu_{d}} \\
& =\sum_{n=1}^{\infty} \sum_{\begin{array}{c}
\mu, \nu \vdash n \\
\mu_{1}, \ldots \mu^{\vdash n} \\
\nu_{1}, \ldots \ldots \nu_{d} \vdash n \\
l\left(\mu_{k}\right), l\left(\nu_{k}\right) \leq N_{k}
\end{array}} \tau^{n} g_{\mu, \mu_{1}, \ldots, \mu_{d}} g_{\nu, \nu_{1}, \ldots, \nu_{d}} \delta_{\mu_{1} \nu_{1}} \cdots \delta_{\mu_{d} \nu_{d}} \delta_{\mu \nu} \\
& =\sum_{n=1}^{\infty} \sum_{\substack{\mu \vdash n \\
\mu_{1}, \ldots \mu_{d} \vdash n \\
l\left(\mu_{k}\right) \leq N_{k}}} \tau^{n} g_{\mu, \mu_{1}, \ldots, \mu_{d}}^{2} \\
& =\sum_{n=1}^{\infty}\left(\sum_{\substack{\mu_{1}, \ldots \mu_{d} \vdash n \\
l\left(\mu_{k}\right) \leq N_{k}}} g_{\mu_{1}, \ldots, \mu_{d}}^{2}\right) \tau^{n},
\end{aligned}
$$

from where we can read that the number of gauge invariants operators is actually counted by the square of the Kronecker coefficients, with the suitable restriction on the permitted partitions for finite $N$. This result is in perfect agreement with the recent direct counting of invariant in tensor models. In the last line of (2.22) we have used the property

$$
\sum_{\mu \vdash n} g_{\mu, \mu_{1}, \ldots, \mu_{d}} g_{\mu, \nu_{1}, \ldots, \nu_{d}}=g_{\mu_{1}, \ldots, \mu_{d}} g_{\nu_{1}, \ldots, \nu_{d}}
$$

when the sum over partitions $\mu$ is not restricted, as can be easily checked by the definition of Kronecker coefficients and the orthogonality relations of characters.

The evaluation of the partition function has been carried out for general tensors with $d$ indices and $G_{d}$ group of symmetry. For simplicity and without loss of generality, we will consider $d=3$ and $G_{3}=\mathrm{U}(N)^{\otimes 3}$ in the rest of the paper.

\section{Kronecker coefficients with a hook shape and multi-matrix models}

Although we do not know any combinatorial formula for computing general Kronecker coefficients, there are some broad families for which we know. The most remarkable of them is perhaps the family of Kronecker coefficients with a hook shape. Started in [47] and refined in [48], this program succeeds in giving $g_{\mu \nu \lambda}$ a combinatorial interpretation when one of the partitions, say $\mu$, is a hook shape. In this section we will use their results to built a compact formula of Kronecker coefficients with a hook shape in terms of Littlewood-Richardson numbers. This formula will allow us to make contact with multi-matrix models, a correspondence that we will show in detail.

Let us consider a hook partition with $n-r$ columns and $r+1$ rows and denote it $\mu(r)$, so $\mu(r)=\left(n-r, 1^{r}\right)$. For $r=0, \ldots, n-1$ the diagram $\mu(r)$ runs over all possible hook shapes. In [48] it was shown that the Kronecker coefficients $g_{\mu(r) \nu \lambda}$ can be expressed in terms of the standard inner products $^{1}$ of Schur and skew Schur functions as

$$
g_{\mu(r) \nu \lambda}+g_{\mu(r-1) \nu \lambda}=\sum_{\gamma \vdash r}\left\langle s_{\lambda}, s_{\nu / \gamma} s_{\gamma^{\prime}}\right\rangle,
$$

\footnotetext{
${ }^{1}$ The standard inner product for symmetric functions is defined as $\left\langle s_{\lambda}, s_{\mu}\right\rangle=\delta_{\lambda \mu}$, where $s_{\lambda}$ and $s_{\mu}$ are Schur functions, see [45].
} 
where $\gamma^{\prime}$ is the $\gamma$-transposed diagram, obtained from $\gamma$ by interchanging rows and columns. We will take eq. (3.1) as the starting point of our analysis.

Using the properties of products of Schur and skew Schur functions

$$
c_{\mu \nu}^{\lambda}=\left\langle s_{\lambda}, s_{\mu} s_{\nu}\right\rangle=\left\langle s_{\lambda / \nu}, s_{\mu}\right\rangle, \quad \lambda \vdash n, \quad \mu \vdash n-r, \quad \nu \vdash r,
$$

where $c_{\mu \nu}^{\lambda}$ are the Littlewood-Richardson numbers, it is only a bit of work to find the compact expression

$$
g_{\mu(r) \nu \lambda}=\sum_{k=0}^{r}(-1)^{r+k} \sum_{\substack{\gamma \vdash k \\ \rho \vdash n-k}} c_{\rho \gamma}^{\nu} c_{\rho \gamma^{\prime}}^{\lambda}, \quad \nu, \lambda \vdash n
$$

where the only partition of 0 is, by definition, $\emptyset$, and $c_{\mu, \emptyset}^{\lambda}=\delta_{\mu}^{\lambda}$. Equation (3.3) shows explicitly the relation between Littlewood-Richardson numbers and Kronecker coefficients with one hook shape and it is completely general for partitions $\nu$ and $\lambda$.

One could ask if eq. (3.3), which involves Kronecker coefficients with 3 partitions, can be generalized to the case of $d$ partitions. This would be very interesting since it would tell about $d$-rank tensor states. Unfortunately, although we believe that a generalization must exist and should reduce to Equation (3.3) for 3 partitions, we do not know it, and it is probably very involved. For instance, if we knew how to express Kronecker coefficients with 4 partitions, one of them being a hook, we could always take the hook to be the one-row partition and obtain the most general expression for the Kronecker coefficients with 3 partitions in terms of Littlewood-Richardson numbers. This is, in fact, an unresolved problem and an important mathematical challenge today.

Equation (3.3) is suggestive from the physical point of view since, as we are going to see, it relates the spectra of energy eigenstates in tensor and matrix theories. As read from eq. (2.22), the spectrum of free tensor models is organized by the Kronecker coefficients, they measure the degeneracy of states (invariant operators) with energy $n$ as

$$
\operatorname{card}\left\{\mathcal{O}_{n}^{G_{3}-\mathrm{Inv}}\right\}=\sum_{\mu, \nu, \lambda \vdash n} g_{\mu \nu \lambda}^{2} .
$$

Thus, $g_{\mu(r) \nu \lambda}^{2}$ has a natural interpretation as counting the hook-shaped sector of the tensor model.

On the other hand, Littlewood-Richardson numbers have been long known to relate to the spectrum of multi-matrix models [35-42]. Specifically, for the case of two different bosonic matrices $Z$ and $Y$

$$
\operatorname{card}\left\{\mathcal{O}_{(n, m)}^{\mathrm{U}(N)-\mathrm{Inv}}\right\}=\sum_{\substack{\mu \vdash n+m \\ \nu \vdash n, \lambda \vdash m}}\left(c_{\nu \lambda}^{\mu}\right)^{2},
$$

where $n$ and $m$ are the number of fields $Z$ and $Y$, respectively, which build the operators. The matrix theory has $\mathrm{U}(N)$ gauge group under which the fields $Z$ and $Y$ transform in the adjoint, so the fields are $N \times N$ matrices. One of the orthogonal basis of operators that relates to this counting is the restricted Schur basis. We will use it from now on. Restricted Schur operators

$$
\chi_{(\mu ; \nu, \lambda) i j}(Z, Y), \quad \mu \vdash n+m, \nu \vdash n, \lambda \vdash m, \quad i, j=1, \ldots, c_{\nu \lambda}^{\mu}
$$

furnish a basis built on $n$ copies of $Z$ and $m$ copies of $Y$. See [37, 38] for details.

Now the question is: in terms of operators (3.6), what is the r.h.s. of eq. (3.3) counting?

First, realize that since $c_{\rho \gamma}^{\nu}$ counts the number of operators $\chi_{(\nu ; \rho, \gamma) i i}(Z, Y)$, it is clear that

$$
\begin{gathered}
\sum_{\substack{\gamma \vdash k \\
\rho \vdash n-k}} c_{\rho \gamma}^{\nu} c_{\rho \gamma^{\prime}}^{\lambda}=\operatorname{card}\left\{\chi_{(\nu ; \rho, \gamma) i i}(Z, Y) \chi_{\left(\lambda ; \rho, \gamma^{\prime}\right) j j}(Z, Y) \mid \gamma \vdash k, \rho \vdash n-k,\right. \\
\left.i=1, \ldots, c_{\rho \gamma}^{\nu}, \quad j=1, \ldots, c_{\rho \gamma^{\prime}}^{\lambda}\right\} .
\end{gathered}
$$


In order to take care of the alternating sum that appears in the r.h.s. of eq. (3.3) we must restrict the set of multi-matrix operators under consideration. Let us first introduce some notation. We will call $\lambda \cap \mu$ the diagram formed from the common boxes of $\mu$ and $\lambda$ as we overlap them. The size of the intersection is always $|\lambda \cap \mu| \leq n$, saturating the inequality when $\lambda=\mu$. A partition is written as $\mu=\left(\mu_{1}, \ldots, \mu_{l(\mu)}\right)$. So, in the language of Young diagrams $\mu_{i}$ is the number of boxes of row $i$, and $l(\mu)$ is the number of rows of the diagram $\mu$. For instance, $\mu_{l(\mu)}$ is the number of boxes of the last row of diagram $\mu$.

It turns out that the alternating sum in the r.h.s. of eq. (3.3) is achieved by restricting $\rho$ to partitions whose last row has the same number of boxes as the last row of $\nu \cap \lambda$, that is,

$$
g_{\mu(r) \nu \lambda}=\sum_{k=0}^{r}(-1)^{r+k} \sum_{\substack{\gamma \vdash k \\ \rho \vdash n-k}} c_{\rho \gamma}^{\nu} c_{\rho \gamma^{\prime}}^{\lambda}=\sum_{\substack{\gamma \vdash r \\ \rho \vdash n-r \\ \rho_{l(\rho)}=(\nu \cap \lambda)_{l(\nu \cap \lambda)}}} c_{\rho \gamma}^{\nu} c_{\rho \gamma^{\prime}}^{\lambda}, \quad \nu, \lambda \vdash n .
$$

Actually, the alternating sum would have also been reproduced if we fix any other corner of $\rho$. The choice of the last row is convenient since the last box of the last row of any Young diagram is always a corner. $^{2}$ With this observation we can write

$$
\begin{array}{r}
g_{\mu(r) \nu \lambda}=\operatorname{card}\left\{\chi_{(\nu ; \rho, \gamma) i i}(Z, Y) \chi_{\left(\lambda ; \rho, \gamma^{\prime}\right) j j}(Z, Y) \mid \gamma \vdash r, \rho \vdash n-r\right. \\
\left.\rho_{l(\rho)}=(\nu \cap \lambda)_{l(\nu \cap \lambda)}, i=1, \ldots, c_{\rho \gamma}^{\nu}, \quad j=1, \ldots, c_{\rho \gamma^{\prime}}^{\lambda}\right\} .
\end{array}
$$

Now, for the sum of squares we have

$$
\begin{aligned}
\sum_{\mu, \lambda \vdash n} g_{\mu(r) \nu \lambda}^{2}= & \operatorname{card}\left\{\chi_{(\nu ; \rho, \gamma) i i}(Z, Y) \chi_{\left(\lambda ; \rho, \gamma^{\prime}\right) j j}(Z, Y) \chi_{\left(\lambda ; \bar{\rho}, \bar{\gamma}^{\prime}\right) k k}^{\dagger}(Z, Y) \chi_{\left(\nu ; \bar{\rho}, \bar{\gamma}^{\prime}\right) l l}^{\dagger}(Z, Y) \mid\right. \\
& \gamma, \bar{\gamma} \vdash r \quad \rho, \bar{\rho} \vdash n-r \quad \nu, \lambda \vdash n \quad \rho_{l(\rho)}=(\nu \cap \lambda)_{l(\nu \cap \lambda)} \\
& \left.i, j=1, \ldots, c_{\rho \gamma}^{\nu} k, l=1, \ldots, c_{\bar{\rho} \bar{\gamma}^{\prime}}^{\lambda}\right\} .
\end{aligned}
$$

Equation (3.11) tells us that the energy spectrum of the hook shape sector labeled by $\mu(r)$ of a 1-boson tensor model is in one-to-one correspondence with the set of rather general composite operators of multi-matrix models shown above. Each observable on the r.h.s. of (3.11) corresponds to the operator that results from the multiplication of the four restricted Schur operators (normal ordered) with the allowed labels. Unfortunately, the OPE for restricted Schur operators is very involved and does not seem to allow for simplifications, see [38] for a detailed discussion. The label $r$ of the hook tells the number of $Y$ fields which enter the operators in the matrix models. For $r=0$, there are only $Z$ fields ( $n$ of them) and $\nu=\lambda$, so the matrix composites are (a power of) Schur polynomials $\chi_{\mu}(Z)$. Now, since the operators $\chi_{\mu}(Z)$ play an important role in $\mathcal{N}=4 \mathrm{SYM}$ for furnishing the $\frac{1}{2}$-BPS sector of the theory [35], and the product of Schur polynomials is a Schur polynomial, we will take license here and call $\left(\chi_{\mu}(Z)\right)^{4} \frac{1}{2}$-BPS operators from now on.

Let us give an example of how the match in equation (3.11) goes.

Example. The simplest while non-trivial example one can take is $n=2$ and $N \geq 2$. In this case, there are two possible values for $r$. It is either $r=0$, and then $\mu(0)=\square$; or $r=1$, with

\footnotetext{
${ }^{2}$ Eq. (3.8) is a sophistication of the identity among combinatorial numbers

$$
\left(\begin{array}{c}
n-1 \\
m
\end{array}\right)=\sum_{k=0}^{m}(-1)^{m+k}\left(\begin{array}{l}
n \\
k
\end{array}\right)
$$


$\mu(1)=\square$. There are two tensor operators that we can form for each $r$, labeled by the partitions $\nu$ and $\lambda$ that make $g_{\mu(r) \nu \lambda}=1$, since for $n=2$ there are no multiplicities. For $r=0$ we have $g_{\square \square, \square, \square}=g_{\square \square, \square \square, \square \square}=1$, whereas for $r=1, g_{\square, \square, \square \square}=g_{\square, \square, \square, \square}=1$. In terms of matrix composites (l.h.s. of (3.11)) we have

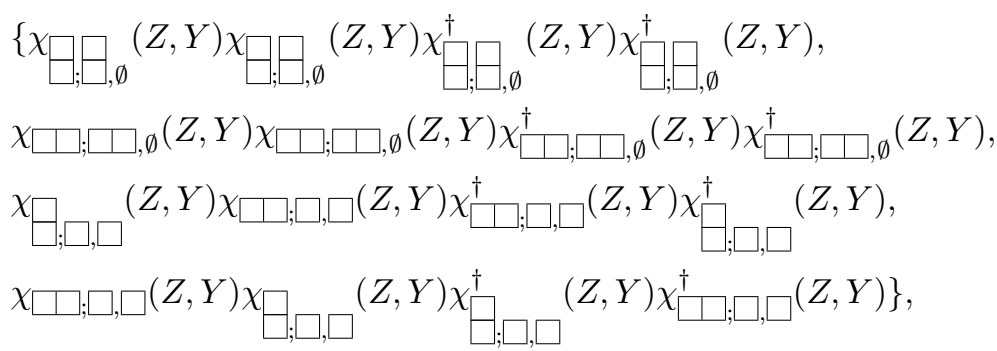

where the first two operators of (3.12) belong to the $r=0$ hook and the last two to $r=1$. Be aware that operators of the form $\chi_{\square ; \square, \square}(Z, Y) \chi_{\square ; \square, \square}(Z, Y) \chi_{\square ; \square, \square}^{\dagger}(Z, Y) \chi_{\square ; \square, \square}^{\dagger} \quad(Z, Y)$ for $r=1$ are not allowed in the set because $\square \cap \square=\square$, and so $\rho_{l(\rho)} \neq(\nu \cap \lambda)_{l(\nu \cap \lambda)}$ since $\rho=\square$. Be also aware that the restricted Schur operators $\chi_{(\lambda ; \rho, \lambda)}(Z, Y)$ are not commuting with each other, so the third and the fourth operators of the set (3.12) are actually different.

What we have done so far is to match the number of eigenstates of both theories in a certain sector. The match is highly non-trivial. Although this does not prove the duality between both theories since we should also match the dynamics of the fields, it clearly tells us that both theories are intimately related. Actually, we will find eq. (3.11) useful in order to interpret the hook sector of the tensor model given that in the context of matrix models restricted Schur operators have a well known meaning. ${ }^{3}$ In order to claim that there is a duality between both theories we should match the dynamics by comparing correlators on both sides. The correlators in the matrix theory are still undefined since we have not spelled out an action for the matrix theory. Although this should be confirmed, a likely scenario is that the correlators of both theories match for a free matrix action of the form $S=Z_{j}^{i} Z_{i}^{\dagger j}+Y_{j}^{i} Y_{i}^{\dagger j}$. In any case, and if this guess doed not work, the question could actually be inverted as to wonder which matrix action makes the correlators match with the free tensor theory. This will be tested in a separate work.

Now, the point here is to understand the meaning of the composite operators that appear in eq. (3.11). For that, let us write the multimatrix composites

$$
\mathcal{O}_{(\rho, \gamma) i j}^{\nu \lambda}=\chi_{(\nu ; \rho, \gamma) i i}(Z, Y) \chi_{\left(\lambda ; \rho, \gamma^{\prime}\right) j j}(Z, Y) \quad \gamma \vdash r, \rho \vdash n-r, \nu, \lambda \vdash n .
$$

With this notation, the operators on the r.h.s. of (3.11) are simply $\mathcal{O}_{(\rho, \gamma) i j}^{\nu \lambda} \mathcal{O}_{(\rho, \gamma) k l}^{\nu \lambda \dagger}$.

First, realize that the operators $\mathcal{O}_{(\rho, \gamma) i j}^{\nu \lambda}$ are 0 if partitions $\nu$ and $\lambda$ differ in more than $r$ boxes, that is, if $|\nu \cap \lambda|<n-r$. As said above, $r=0$ forces $\lambda=\nu$. Let us think of the operators $\mathcal{O}^{\nu \nu}=\chi_{\nu}(Z) \chi_{\nu}(Z)$ for $r=0$ as the initial (unperturbed) states and consider the operators with $r=1,2, \ldots$ as fluctuations of those states with increasing energy. We will interpret the parameter $r$ as the depth of the fluctuation. Thus, for $r=1$, for which $|\nu \cap \lambda| \geq n-1$, the operator ${ }^{4} \mathcal{O}_{(\rho,(1))}^{\nu \lambda}$ will encode a 1-box fluctuation of the state $\nu$ into $\lambda$, making explicit the transition state $\rho \vdash n-1$. The same applies for subsequent values of $r$, where the state $\nu$ turns into $\lambda$ after an $r$-box fluctuation. Be aware that the process is symmetric, so the role of $\nu$ might have also been taken by $\lambda$.

\footnotetext{
${ }^{3}$ Especially in the displaced corner approximation [49-53], where restricted Schur operators have been proven to be holographically dual to giant gravitons with strings attached.

${ }^{4}$ Note that for $r=0,1,2$ there are no multiplicities, so the latin indices are absent.
} 
In summary, the operators (3.13) (and so (3.11)) seem to give a complete description of the possible $Y$-driven fluctuations of $\frac{1}{2}$-BPS states in the matrix theory.

We would like to briefly comment on the case where $G_{3}=\mathrm{U}(1) \otimes \mathrm{U}\left(N_{2}\right) \otimes \mathrm{U}\left(N_{3}\right)$, that is, when $N_{1}=1$. Now, the only irrep of $\mathrm{U}(1)$ with $n$ boxes is one-dimensional and labeled by the row $(n)$. This forces $r=0$, and $\mu(0)=(n)$. The Kronecker coefficients turn out to be

$$
g_{(n) \nu \lambda}=\frac{1}{n !} \sum_{\sigma \in S_{n}} \chi_{(n)}(\sigma) \chi_{\nu}(\sigma) \chi_{\lambda}(\sigma)=\frac{1}{n !} \sum_{\sigma \in S_{n}} \chi_{\nu}(\sigma) \chi_{\lambda}(\sigma)=\delta_{\nu \lambda}
$$

From the tensor point of view, there is just one invariant operator for each partition $\lambda$. This operator is related with the matrix operator $\chi_{\lambda}^{2}(Z) \chi_{\lambda}^{\dagger 2}(Z)$, which, as a multiplication of Schurs, is a Schur operator. The tensor theory with two indices matches the 1-matrix theory, and is suitable to describe the 1/2-BPS sector. It seems that tensor theories of rank $d$ should be related with multi-matrix theories with $d-1$ especies. We conjecture that this is indeed the case.

\section{Hagedorn phase transition for finite $N$}

Recently, It has been noticed that tensor models (of any classical gauge group) present such a rapid growth of states that there is no Hagedron behaviour in the limit $N \rightarrow \infty[54,55]$. Actually, the partition function (2.22) is not even convergent for any finite value of $\tau$. This is because, as noticed in $[1,43]$,

$$
X_{\infty}(n) \equiv \sum_{\mu, \lambda, \nu \vdash n} g_{\mu \nu \lambda}^{2}=\sum_{\lambda \vdash n} z_{\lambda},
$$

and we know that

$$
\lim _{n \rightarrow \infty} \frac{\sum_{\lambda \vdash n} z_{\lambda}}{n !}=1
$$

Actually the convergence of (4.2) is quite fast. The reason for it is that the sum is dominated by the term associated with the one column Young diagram $z_{\left(1^{n}\right)}=n$ !, the rest of the terms are subleading. Now, since $\tau$ is physically related to the temperature through $\tau=e^{-1 / k_{B} T}$, the zeroradius convergence of the partition function series for $N \rightarrow \infty$ can be understood as the Hagedorn temperature going to 0 at that limit.

However, for finite $N$ the spectrum gets truncated since no states for which $l\left(\mu_{k}\right)>N$ are allowed. Actually, for finite $N$ the number of states is given by

$$
X_{N}(n) \equiv \sum_{\substack{\mu_{1}, \mu_{2}, \mu_{3} \vdash n \\ l\left(\mu_{k}\right) \leq N}} g_{\mu_{1} \mu_{2} \mu_{3}}^{2},
$$

as can be read from (2.22).

The growth of $X_{N}(n)$ is then exponential at large $n$ and the system is expected to present Hagedorn behaviour with a temperature

$$
T_{H}(N) \sim \frac{1}{\log N},
$$

as noticed in [54]. As usual, Hagedorn behaviour indicates the existence of a phase transition at $T_{H}$. So, if we start with a low energy state and we pump energy into the system the second phase will appear at some point. The two phases will coexist from then on and the temperature will asymptotically stabilize at $T_{H}$. The partition function, which below $T_{H}$ is summable, describes one of the two phases. In this section we will argue that the phase that arises at high energy (whose states are not accounted in $\left.Z_{s}(\tau)\right)$ can be interpreted as a fluctuating $\frac{1}{2}$-BPS state, in a similar fashion as we treated the hook sector of the tensor model in the former section. 
To support this claim we will give evidence that the number of invariant $n$-fold operators that are "left out" in $Z_{s}(\tau)$ for finite $N$ when $N<n$, namely $X_{\infty}(n)-X_{N}(n)$, possibly match the number of fluctuations of $\frac{1}{2}$-BPS states, when the energy of the fluctuations (depth) is given by $n^{\prime}=n-N-1$. We say "possibly match" since we will not be able to compute $X_{N}(n)$ exactly for $n^{\prime} \geq 2$.

Unperturbed $\frac{\mathbf{1}}{\mathbf{2}}$-BPS state. Let us consider $n=N+1$ first, so $n^{\prime}=0$, which is the energy at which the second phase is expected to appear. The number of states which are "left out" can be calculated exactly in this case. Notice, that these states must be labeled by three partitions where one should be the one-column, let us take it to be $\mu$, so $\mu=\left(1^{n}\right)$. In this case the Kronecker coefficients are easily calculated from the orthogonality properties of characters as

$$
g_{\left(1^{n}\right) \nu \lambda}=\frac{1}{n !} \sum_{\sigma \in S_{n}} \chi_{\left(1^{n}\right)}(\sigma) \chi_{\nu}(\sigma) \chi_{\lambda}(\sigma)=\frac{1}{n !} \sum_{\sigma \in S_{n}} \chi_{\nu^{\prime}}(\sigma) \chi_{\lambda}(\sigma)=\delta_{\nu^{\prime} \lambda},
$$

where $\nu^{\prime}$ is the transposed diagram of $\nu$. Now, the number of states which are left out is

$$
X_{\infty}(N+1)-X_{N}(N+1)=\sum_{\nu, \lambda \vdash N+1} g_{\left(1^{N+1}\right) \nu \lambda}^{2}=\sum_{\nu, \lambda \vdash N+1} \delta_{\nu^{\prime} \lambda}=P_{N+1} .
$$

So, at the threshold energy $n=N+1$, the tensor model in its second phase presents the degeneracy of (unexcited) $\frac{1}{2}$-BPS states labeled by $\nu \vdash n$.

Single fluctuation of the $\frac{1}{2}$-BPS state. If we keep on pumping energy into the system, part of it will go into exciting modes labeled by partitions whose number of rows do not exceed $N$, and part of the energy will go to the second phase, exciting the $\frac{1}{2}$-BPS state. Let us take $n=N+2$, so $n^{\prime}=1$. The states which are associated to the second phase must have $\mu=\left(1^{N+2}\right)$ or $\mu=\left(2,1^{N}\right)$. For the first option the counting follows the same path as before leading to a total of $P_{N+2}$ states. The number of states that correspond to $\mu=\left(2,1^{N}\right)$ can be calculated exactly, since the partition $\left(2,1^{N}\right)$ is a hook. ${ }^{5}$ They match the number of operators $\mathcal{O}_{(\rho,(1))}^{\nu^{\prime} \lambda}$ which, as seen before, are interpreted as 1-box fluctuations of the $\frac{1}{2}$-BPS state labeled by $\nu^{\prime}$.

b, Small fluctuations of the $\frac{1}{2}$-BPS state. For $n^{\prime} \geq 2$ the relevant states of the second phase are labeled by a partition $\mu$, made of one column of $N+1$ boxes and a diagram $\alpha$ with $n^{\prime}$ boxes attached to the column (as shown in the figure), along with diagrams $\nu^{\prime}, \lambda \vdash n$. Note that for $n^{\prime} \geq 2$ the Kronecker coefficients get harder to compute exactly. For instance, if $n^{\prime}=2$ we do not have yet a combinatorial method to compute Kronecker coefficients of the type $g_{\left(2^{2}, 1^{N-1}\right) \nu^{\prime} \lambda}$. In order to estimate those Kronecker coefficients we will use the following result:

Theorem ([56-58]). Let $\mu, \nu, \lambda \vdash n$, and denote $n^{\prime}=n-\mu_{1}$, where $\mu_{1}$ refers to the first row of $\mu$. Now, if $n^{\prime}<n-|\nu \cap \lambda|$ then $g_{\mu \nu \lambda}=0$.

This statement naturally applies to the cases we are considering in this section, with $\mu$ as in the figure, so $n^{\prime}=|\alpha|$. Actually, although the result uses the first row of the diagram $\mu$ we can translate it into the first column of $\mu$ by changing $\mu \rightarrow \mu^{\prime}$ and $\nu \rightarrow \nu^{\prime}$, since $g_{\mu \nu \lambda}=g_{\mu^{\prime} \nu^{\prime} \lambda}$. The theorem clearly holds for hook shapes, but the usefulness of it relies on its application for general shapes $\mu$. In particular, for the partitions $\mu$ we are considering, the theorem reinforces the interpretation of $n^{\prime}=|\alpha|$ being the depth of the fluctuation. So, as for hook shapes, we will interpret the tensor states counted by $g_{\mu \nu^{\prime} \lambda}$ with $\mu$ as in the figure as $n^{\prime}$-depth fluctuations of $\frac{1}{2}$-BPS states in matrix models labeled by $\nu^{\prime}$.

\footnotetext{
${ }^{5}$ The partition $\mu=\left(2,1^{N}\right)$ corresponds to $r=N$ with the convention we are using. Now, since $g_{\mu \nu \lambda}=g_{\mu^{\prime} \nu^{\prime} \lambda}$, as can be checked from the definition, $g_{\mu(N) \nu \lambda}=g_{\mu(1) \nu^{\prime} \lambda}$.
} 


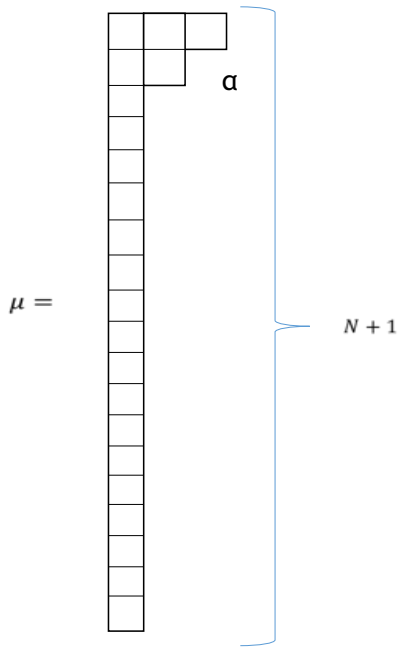

Figure 1. Typical Young diagram for states of the second phase. Here $|\alpha|=n^{\prime}$.

It is likely that there exist combinatorial formulas for $g_{\mu \nu \lambda}$ similar to (3.3), a result that would be extremely interesting in this discussion. Probably, the constraint $\rho_{l(\rho)}=(\nu \cap \lambda)_{l(\nu \cap \lambda)}$ that appears in (3.11) (which is related to a 1-row diagram $\alpha$ ) should be replaced by any other related to a more general shape of $\mu$. We conjecture that this is actually the case and so the fluctuations are described by operators (3.13) with specific constraints for $\rho$ and $\nu \cap \lambda$ related to $\mu$. This way the second phase of the tensor model would be described by a multi-matrix model.

To finish this section we will offer an estimation of the total number of states of the second phase for large $N$ and $n^{\prime} \ll N$. We will use the conjecture above-mentioned and the corner approximations obtained in the appendix. The total number of states, calculated from (A.9) and (A.10), is

$$
X_{\infty}\left(N+n^{\prime}+1\right)-X_{N}\left(N+n^{\prime}+1\right) \sim a_{n^{\prime}} P_{N} N^{n^{\prime}}
$$

where $a_{n^{\prime}} \sim 2 n^{\prime}+1$ seems to hold. ${ }^{6}$

\section{Summary and outlook}

We have started the paper by writing and evaluating the partition function of free color tensor models with a symmetry group $G_{d}$. The partition function shows that the spectrum of $G_{d}$-invariant energy eigenstates is organized by Kronecker coefficients, an expected result which serves as a consistency check. Then, using recent mathematical algorithms for computing Kronecker coefficients with a hook shape we have derived eq. (3.3). This identity has not appear in the literature before as far as we know and shows that, in the hook sector, Kronecker coefficients are computed by Littlewood-Richardson numbers. Now, the Littlewood-Richardson numbers are known to organize the spectrum of multi-matrix models. So, we step forward and interpret (3.3) as relating both spectra of the theories in certain sectors. A precise match of the multi-matrix sector and a tensor hook shaped sector is shown in eq. (3.11), which is the main result of the paper. Eq. (3.11) shows that for certain energy level determined by $n$, the different tensor states with a hook diagram $\mu(r)$ can be matched one-to-one with fluctuations driven by $r Y$ fields of a Schur polynomial $\chi_{\mu}(Z)$ in

\footnotetext{
${ }^{6}$ Numerical computations upto $n=100$ and $n^{\prime}=7$ show a good agreement with these values of $a_{n^{\prime}}$.
} 
the multi-matrix model. This strongly suggests that the 1-bosonic tensor model contains a multimatrix model with two different species $Z$ and $Y$, each tensor state encoding a fluctuation of the Schur polynomial state. The results so far apply to the hook sector of the tensor model.

One can see from the form of the partition function (2.22) that the partition function is not summable for $N \rightarrow \infty$, but it grows exponentially for finite $N$. This growth is a sign of a Hagedorn phase transition. Using known results for Kronecker coefficients we conjecture that the high energy Hagedorn phase, which appears at $n=N+n^{\prime}+1$ for $n^{\prime}=0,1, \ldots$ could be described by a multi-matrix theory and interpreted again as fluctuations of depth $n^{\prime}$ of Schur polynomial states.

Given the match between spectra of both theories in the hook sector, a natural question is: can we find a dynamical equivalence of both theories in the hook sector? This will be especially interesting for the interacting theory. Remember that the $\mathrm{SU}(2)$ sector of $\mathcal{N}=4 \mathrm{SYM}$ is described by operators built on two matrix species $Z$ and $Y$, and that it is hard to tackle perturbatively. However, perturbative tensor models with a quartic interaction are known to lead to melonic Feynman diagrams which are much easier to handle.

It will be interesting to investigate the conjecture that the second phase, which appears at energies $n=N+n^{\prime}+1$ for $n^{\prime}=0,1, \ldots$, is described by a multi-matrix theory with two species $Z$ and $Y$. We have conjectured that tensor states of the second phase correspond to fluctuations of Schur polynomial with depth $n^{\prime}$. So the idea is to find the constraint on fluctuations for general shapes $\alpha$ analogous to $\rho_{l(\rho)}=(\nu \cap \lambda)_{l(\nu \cap \lambda)}$ that appears in (3.11) for hook shapes. This is surely a tough problem, since finding the general rule would shed light on how to compute Kronecker coefficients using combinatorics, a mathematical problem which is lacking for a solution since 80 years ago.

More generally, it will be desirable to investigate the relation between tensor and matrix models at the level of their actions. For instance, could matrix models appear as tensor model effective theories? This would clarify, for instance, if the flirt that we have shown in this paper is actually the beginning of a long term affair.

\section{Acknowledgments}

I thank S. Das, R. de Mello Koch, S-J. Rey, A. Rosabal and M. Walton for useful discussions. This work was partly supported by the Natural Sciences and Engineering Research Council of Canada, the University of Lethbridge, and by the Institute of Basic Science (South Korea) IBS-R018-D2.

\section{A Kronecker coefficients with a hook shape: corner approximation}

In order to make estimations of $g_{\mu(r) \nu \lambda}^{2}$ and the sums which count the number of states, we will find useful to use the number of corners of a diagram, $C(\rho)$, which measures the number of boxes that can be deleted from diagram $\rho$ and still lead to a valid Young diagram. In the language of partitions, the number of corners is the number of different parts in partition $\rho$. Thus, in this appendix we will arrive at approximations of (3.3) for which we only use the number of corners of the diagrams. We will trust these approximation in the regime of large $N$ and $n^{\prime} \ll N$, in which case they are expected to reproduce the leading order (in $1 / N$ ) correctly.

Case $\boldsymbol{r}=1$. For the simplest non-trivial case, $r=1$, we will have $\mu(1)=(n-1,1)$ which is the transpose of $\mu(n-2)=\left(2,1^{n-2}\right)$. From the definition of Kronecker coefficients we immediately see that $g_{\mu(1) \nu \lambda}=g_{\mu(n-2) \nu^{\prime} \lambda}$. With a diagram $\mu=\left(2,1^{n-2}\right)$ we can find an exact formula for the sum of the square of Kronecker coefficients. A formula which involves only corners of partitions.

First, let us take two separate cases depending on whether the other two diagrams $\nu^{\prime}$ and $\lambda$ are equal or not. If $\nu^{\prime} \neq \lambda$, where $\nu^{\prime}$ is the transposed diagram of $\nu$, formula (3.3) tells us that 
the Kronecker coefficient will be one if diagram $\lambda$ is obtained by taking a corner box from $\nu^{\prime}$ and return it somewhere else. Otherwise it is zero. We can count all the possible non-zero combinations in the following way. Let us pick a diagram $\rho \vdash n-1$. The statement that $\rho$ is connected (in the branching graph) with $\nu^{\prime}$ and $\lambda$ means that adding a box to $\rho$ at one of its internal corners produces $\nu^{\prime}$ and adding a box at a different internal corner produces $\lambda$. Now, if $\nu^{\prime}$ and $\lambda$ are connected then the Kronecker coefficient $g_{\left(2,1^{n-2}\right) \nu^{\prime} \lambda}$ is 1 . Given two different diagrams $\nu^{\prime}, \lambda \vdash n$ there is a unique $\rho \vdash n-1$ such that $\rho=\nu^{\prime} \cap \lambda$. So, all the connected combinations $\left(\nu^{\prime}, \lambda\right)$ are found if we consider all diagrams $\rho \vdash n-1$, and for each one all possible ways of attaching a pair of boxes, that is,

$$
\sum_{\nu^{\prime} \neq \lambda} g_{\left(2,1^{n-2}\right) \nu^{\prime} \lambda}^{2}=\sum_{\nu^{\prime} \neq \lambda} g_{\left(2,1^{n-2}\right) \nu^{\prime} \lambda}=\sum_{\rho \vdash n-1} C(\rho)(C(\rho)+1),
$$

where $C(\rho)$ is the number of corners of diagram $\rho$. In the first equality of (A.1) we have used the fact that $g_{\left(2,1^{n-2}\right) \nu^{\prime} \lambda}$ is either 0 or 1 .

When $\nu^{\prime}=\lambda$ we can read from (3.3) that

$$
g_{\left(2,1^{n-2}\right) \nu^{\prime} \nu}=C(\nu)-1 .
$$

Gluing (A.1) and (A.2) we obtain

$$
\sum_{\nu, \lambda \vdash n} g_{\left(2,1^{n-2}\right) \nu^{\prime} \lambda}^{2}=\sum_{\rho \vdash n-1} C(\rho)(C(\rho)+1)+\sum_{\rho \vdash n}(C(\rho)-1)^{2} .
$$

This is an exact formula.

Case $\boldsymbol{r}=\mathbf{2}$. In (3.3) we can see that the computation of the Kronecker coefficients for $r=2$, or equivalently for $r=N$ (our case) involves, at the most, diagrams with two boxes for $\gamma$. It is known that the only values that the Littlewood-Richardson numbers can take when one of the diagrams has two boxes or less are 0 or 1 . So in this case we should not worry about multiplicities either. However, for the case $r=2$ it will not be possible to find an exact formula in terms of corners as we have done for $r=1$. For an exact formula we would need more information about the diagrams than just corners, like the number of double corners, which corresponds to rows from which we could remove two boxes and still arrive at a valid Young diagram. Nevertheless, we can make an estimation of the order based on the number of corners.

First, realize that in (3.3) the highest power of corners will happen when $k=2$ and it will be 4. For $k=1$ we saw in the paragraph above that the highest power was 2 . In general, the highest power of corners in the sum will appear for $k=r$ and it will be $2 r$. Now, since for large $N$ the sum will be clearly dominated by terms which involve the highest power of corners, for $r=2$ we will consider only $k=2$ bellow, and so $\gamma \vdash 2$ in (3.3).

Now, for $\gamma \vdash 2$, the product $c_{\rho \gamma}^{\nu^{\prime}} c_{\rho \gamma^{\prime}}^{\lambda}$ will be 1 if deleting two boxes from $\nu^{\prime}$ and gluing them at internal corners results in $\lambda$, provided that if the boxes deleted are in the same row (column) of $\nu^{\prime}$ they are not in the same row (column) of $\lambda$. Otherwise the product will be 0 . We will not consider the cases where the boxes are deleted or placed at the same row/column. This restriction will allow us to still obtain the leading order at large $N$ using only the corners of the diagrams in our estimations. The number of pairs $\left(\nu^{\prime}, \lambda\right)$ which are left out with this restriction are not many. They are actually negligible for large $N$ since for diagrams with a large number of corners the number of choices of deleting (and gluing) two boxes at different places is much higher than the number of choices at the same row. So contributions from deleting/placing boxes at the same row/column will be always subleading.

We will distinguish three cases depending on $\left|\nu^{\prime} \cap \lambda\right|$ being $n, n-1$ or $n-2$. 
If $\left|\nu^{\prime} \cap \lambda\right|=n$ then $\nu^{\prime}=\lambda$. In this case we have $g_{\left(3,1^{n-3}\right) \lambda^{\prime} \lambda} \sim 2\left(\begin{array}{c}C(\lambda) \\ 2\end{array}\right)$, so

$$
\sum_{\rho \vdash n} g_{\left(3,1^{n-3}\right) \rho^{\prime} \rho}^{2} \sim \sum_{\rho \vdash n} 4\left(\begin{array}{c}
C(\rho) \\
2
\end{array}\right)^{2} .
$$

If $\nu^{\prime} \cap \lambda=\rho \vdash n-1$, so the diagrams differ in one box, then $g_{\left(3,1^{n-3}\right) \nu^{\prime} \lambda} \sim 2 C\left(\nu^{\prime} \cap \lambda\right)$. So

$$
\sum_{\nu^{\prime} \cap \lambda \vdash n-1} g_{\left(3,1^{n-3}\right) \nu^{\prime} \lambda}^{2} \sim \sum_{\rho \vdash n-1} 4 C(\rho)^{2} C(\rho)(C(\rho)+1) .
$$

If $\nu^{\prime} \cap \lambda=\rho \vdash n-2$, so the diagrams differ in two boxes, then $g_{\left(3,1^{n-3}\right) \nu^{\prime} \lambda}=2$, where 2 comes from the sum over $\gamma \vdash 2$, and

$$
\sum_{\nu^{\prime} \cap \lambda \vdash n-2} g_{\left(3,1^{n-3}\right) \nu^{\prime} \lambda}^{2} \sim \sum_{\rho \vdash n-2} 4\left(\begin{array}{c}
C_{i}(\rho) \\
2
\end{array}\right)\left(\begin{array}{c}
C_{i}(\rho)-2 \\
2
\end{array}\right) \sim \sum_{\rho \vdash n-2} 4\left(\begin{array}{c}
C(\rho)+1 \\
2
\end{array}\right)\left(\begin{array}{c}
C(\rho)-1 \\
2
\end{array}\right) .
$$

Consistently, we will take into account the sums of the contributions coming from $C(\rho)^{4}$. From (A.4), (A.5) and (A.6) we see that

$$
\sum_{\nu, \lambda \vdash n} g_{\left(3,1^{n-3}\right) \nu \lambda}^{2} \sim 6 \sum_{\rho \vdash n} C(\rho)^{4},
$$

where we have taken into account that

$$
\frac{\sum_{\rho \vdash n-n^{\prime}} C(\rho)^{r}}{\sum_{\rho \vdash n} C(\rho)^{r}} \longrightarrow 1, \quad n \rightarrow \infty .
$$

General $\boldsymbol{r}=\boldsymbol{n}^{\prime}$. When $n=N+n^{\prime}+1$ with $n^{\prime} \ll N$ we will be interested in calculating sums of $g_{\left(n^{\prime}+1,1^{N}\right) \nu^{\prime} \lambda}^{2}$. When we estimate the sums using corners we will have terms in the sum like $C(\rho)^{2 n^{\prime}}$ which will dominate the sum. So we will consider those terms only. Also, the approaches taken for the case $r=2$ will apply here. Be aware that all these approximations make sense only for large $N$ and $n^{\prime} \ll N$. Notice that for the cases we consider in these approximations where $n^{\prime}$ boxes are deleted from $\nu^{\prime}$ at different corners, the Littlewood-Richardson numbers are $c_{\rho \gamma}^{\nu^{\prime}}=d_{\gamma}$, where $d_{\gamma}$ is the dimension of the representation of the symmetric group labeled by partition $\gamma$. So $c_{\rho \gamma}^{\nu^{\prime}} c_{\rho \gamma}^{\nu^{\prime}}=d_{\gamma}^{2}$. Now, the sum in $\gamma$ that appears in (3.3) can be performed to give a factor $n^{\prime}$ !, since $\sum_{\gamma \vdash n^{\prime}} d_{\gamma}^{2}=n^{\prime}$ !.

All in all, the total sum can be approximated (to leading term) as

$$
\sum_{\nu^{\prime}, \lambda \vdash N+n^{\prime}+1} g_{\left(n^{\prime}+1,1^{N}\right) \nu^{\prime} \lambda}^{2} \sim A\left(n^{\prime}\right) \sum_{\rho \vdash n} C(\rho)^{2 n^{\prime}},
$$

with

$$
A\left(n^{\prime}\right)=n^{\prime} !^{2} \sum_{m=0}^{n^{\prime}}\left(\begin{array}{l}
n^{\prime} \\
m
\end{array}\right)^{2}\left(\begin{array}{c}
n^{\prime} \\
n^{\prime}-m
\end{array}\right)^{2}
$$

Open Access. This article is distributed under the terms of the Creative Commons Attribution License (CC-BY 4.0), which permits any use, distribution and reproduction in any medium, provided the original author(s) and source are credited.

\section{References}

[1] M.W. Hero and J.F. Willenbring, Stable Hilbert series as related to the measurement of quantum entanglement, Discrete Math. 309 (2009) 6508.

[2] M. Christandl and G. Mitchison, The spectra of quantum states and the Kronecker coefficients of the symmetric group, Commun. Math. Phys. 261 (2006) 789 [quant-ph/0409016].

[3] P. Di Francesco, P.H. Ginsparg and J. Zinn-Justin, 2-D Gravity and random matrices, Phys. Rept. 254 (1995) 1 [hep-th/9306153] [INSPIRE]. 
[4] J. Ambjørn, B. Durhuus and T. Jonsson, Three-dimensional simplicial quantum gravity and generalized matrix models, Mod. Phys. Lett. A 6 (1991) 1133 [InSPIRE].

[5] M. Gross, Tensor models and simplicial quantum gravity in $>$ 2-D, Nucl. Phys. Proc. Suppl. 25A (1992) 144 [INSPIRE].

[6] N. Sasakura, Tensor model for gravity and orientability of manifold, Mod. Phys. Lett. A 6 (1991) 2613 [INSPIRE].

[7] S. Sachdev and J. Ye, Gapless spin fluid ground state in a random, quantum Heisenberg magnet, Phys. Rev. Lett. 70 (1993) 3339 [cond-mat/9212030] [INSPIRE].

[8] A. Kitaev, Hidden correlations in the Hawking radiation and thermal noise, talks at KITP Strings Seminar and Entanglement 2015 program, February 12, April 7, and May 27, 2015.

[9] J. Polchinski and V. Rosenhaus, The Spectrum in the Sachdev-Ye-Kitaev Model, JHEP 04 (2016) 001 [arXiv: 1601.06768] [INSPIRE].

[10] A. Jevicki, K. Suzuki and J. Yoon, Bi-Local Holography in the SYK Model, JHEP 07 (2016) 007 [arXiv: 1603.06246] [INSPIRE].

[11] J. Maldacena and D. Stanford, Remarks on the Sachdev-Ye-Kitaev model, Phys. Rev. D 94 (2016) 106002 [arXiv: 1604.07818] [INSPIRE].

[12] S.R. Das, A. Jevicki and K. Suzuki, Three Dimensional View of the SYK/AdS Duality, JHEP 09 (2017) 017 [arXiv: 1704.07208] [INSPIRE].

[13] S.R. Das, A. Ghosh, A. Jevicki and K. Suzuki, Space-Time in the SYK Model, arXiv:1712.02725 [INSPIRE].

[14] S.R. Das, A. Ghosh, A. Jevicki and K. Suzuki, Three Dimensional View of Arbitrary q SYK models, JHEP 02 (2018) 162 [arXiv:1711.09839] [INSPIRE].

[15] J. Yoon, SYK Models and SYK-like Tensor Models with Global Symmetry, JHEP 10 (2017) 183 [arXiv: 1707.01740] [INSPIRE].

[16] P. Narayan and J. Yoon, Supersymmetric SYK Model with Global Symmetry, arXiv: 1712.02647 [INSPIRE].

[17] T. Azeyanagi, F. Ferrari and F.I. Schaposnik Massolo, Phase Diagram of Planar Matrix Quantum Mechanics, Tensor and Sachdev-Ye-Kitaev Models, Phys. Rev. Lett. 120 (2018) 061602 [arXiv: 1707.03431] [INSPIRE].

[18] E. Witten, An SYK-Like Model Without Disorder, arXiv:1610.09758 [INSPIRE].

[19] R. Gurau, Colored Group Field Theory, Commun. Math. Phys. 304 (2011) 69 [arXiv: 0907.2582] [INSPIRE].

[20] R. Gurau and J.P. Ryan, Colored Tensor Models - a review, SIGMA 8 (2012) 020 [arXiv:1109.4812] [INSPIRE].

[21] H. Itoyama, A. Mironov and A. Morozov, Ward identities and combinatorics of rainbow tensor models, JHEP 06 (2017) 115 [arXiv:1704.08648] [INSPIRE].

[22] H. Itoyama, A. Mironov and A. Morozov, Rainbow tensor model with enhanced symmetry and extreme melonic dominance, Phys. Lett. B 771 (2017) 180 [arXiv:1703.04983] [INSPIRE].

[23] A. Tanasa, Multi-orientable Group Field Theory, J. Phys. A 45 (2012) 165401 [arXiv:1109.0694] [INSPIRE]. 
[24] R. Gurau, The 1/N expansion of colored tensor models, Annales Henri Poincaré 12 (2011) 829 [arXiv: 1011.2726] [INSPIRE].

[25] R. Gurau and V. Rivasseau, The 1/N expansion of colored tensor models in arbitrary dimension, EPL 95 (2011) 50004 [arXiv:1101.4182] [INSPIRE].

[26] R. Gurau, The complete $1 / N$ expansion of colored tensor models in arbitrary dimension, Annales Henri Poincaré 13 (2012) 399 [arXiv:1102.5759] [INSPIRE].

[27] R. Gurau, The complete $1 / N$ expansion of a SYK-like tensor model, Nucl. Phys. B 916 (2017) 386 [arXiv: 1611.04032] [INSPIRE].

[28] S. Dartois, V. Rivasseau and A. Tanasa, The $1 / N$ expansion of multi-orientable random tensor models, Annales Henri Poincaré 15 (2014) 965 [arXiv:1301.1535] [INSPIRE].

[29] H. Itoyama, A. Mironov and A. Morozov, Cut and join operator ring in tensor models, Nucl. Phys. B 932 (2018) 52 [arXiv:1710.10027] [INSPIRE].

[30] P. Diaz and S.-J. Rey, Orthogonal Bases of Invariants in Tensor Models, JHEP 02 (2018) 089 [arXiv: 1706.02667 ] [INSPIRE].

[31] R. de Mello Koch, R. Mello Koch, D. Gossman and L. Tribelhorn, Gauge Invariants, Correlators and Holography in Bosonic and Fermionic Tensor Models, JHEP 09 (2017) 011 [arXiv: 1707.01455] [INSPIRE].

[32] J. Ben Geloun and S. Ramgoolam, Tensor Models, Kronecker coefficients and Permutation Centralizer Algebras, JHEP 11 (2017) 092 [arXiv:1708.03524] [INSPIRE].

[33] P. Diaz and S.-J. Rey, Invariant Operators, Orthogonal Bases and Correlators in General Tensor Models, Nucl. Phys. B 932 (2018) 254 [arXiv:1801.10506] [INSPIRE].

[34] A. Mironov and A. Morozov, Correlators in tensor models from character calculus, Phys. Lett. B 774 (2017) 210 [arXiv:1706.03667] [INSPIRE].

[35] S. Corley, A. Jevicki and S. Ramgoolam, Exact correlators of giant gravitons from dual $N=4$ SYM theory, Adv. Theor. Math. Phys. 5 (2002) 809 [hep-th/0111222] [INSPIRE].

[36] V. Balasubramanian, D. Berenstein, B. Feng and M.-x. Huang, D-branes in Yang-Mills theory and emergent gauge symmetry, JHEP 03 (2005) 006 [hep-th/0411205] [INSPIRE].

[37] R. Bhattacharyya, S. Collins and R. de Mello Koch, Exact Multi-Matrix Correlators, JHEP 03 (2008) 044 [arXiv: 0801.2061] [INSPIRE].

[38] R. Bhattacharyya, R. de Mello Koch and M. Stephanou, Exact Multi-Restricted Schur Polynomial Correlators, JHEP 06 (2008) 101 [arXiv:0805.3025] [INSPIRE].

[39] Y. Kimura and S. Ramgoolam, Branes, anti-branes and brauer algebras in gauge-gravity duality, JHEP 11 (2007) 078 [arXiv:0709.2158] [INSPIRE].

[40] Y. Kimura, Non-holomorphic multi-matrix gauge invariant operators based on Brauer algebra, JHEP 12 (2009) 044 [arXiv:0910.2170] [INSPIRE].

[41] T.W. Brown, P.J. Heslop and S. Ramgoolam, Diagonal multi-matrix correlators and BPS operators in $N=4$ SYM, JHEP 02 (2008) 030 [arXiv:0711.0176] [INSPIRE].

[42] T.W. Brown, P.J. Heslop and S. Ramgoolam, Diagonal free field matrix correlators, global symmetries and giant gravitons, JHEP 04 (2009) 089 [arXiv:0806.1911] [INSPIRE].

[43] J. Ben Geloun and S. Ramgoolam, Counting Tensor Model Observables and Branched Covers of the 2-Sphere, arXiv:1307.6490 [INSPIRE]. 
[44] B.-S. Skagerstam, On the Large $N_{c}$ Limit of the $\mathrm{SU}\left(N_{c}\right)$ Colour Quark-Gluon Partition Function, Z. Phys. C 24 (1984) 97.

[45] I.G. MacDonald, Symmetric Functions and Hall Polynomials, second editon, Oxford University Press, Oxford (1995).

[46] F.A. Dolan, Counting BPS operators in $N=4$ SYM, Nucl. Phys. B 790 (2008) 432 [arXiv:0704.1038] [INSPIRE].

[47] J. Blasiak, Kronecker coefficients for one hook shape, arXiv:1209.2018.

[48] R. Liu, A simplified Kronecker rule for one hook shape, Proc. Amer. Math. Soc. 145 (2017) 3657 [arXiv: 1412.2180].

[49] R. de Mello Koch, J. Smolic and M. Smolic, Giant Gravitons - with Strings Attached (I), JHEP 06 (2007) 074 [hep-th/0701066] [INSPIRE].

[50] R. de Mello Koch, J. Smolic and M. Smolic, Giant Gravitons - with Strings Attached (II), JHEP 09 (2007) 049 [hep-th/0701067] [INSPIRE].

[51] D. Bekker, R. de Mello Koch and M. Stephanou, Giant Gravitons - with Strings Attached. III., JHEP 02 (2008) 029 [arXiv:0710.5372] [INSPIRE].

[52] R. de Mello Koch, G. Mashile and N. Park, Emergent Threebrane Lattices, Phys. Rev. D 81 (2010) 106009 [arXiv: 1004.1108] [INSPIRE].

[53] V. De Comarmond, R. de Mello Koch and K. Jefferies, Surprisingly Simple Spectra, JHEP 02 (2011) 006 [arXiv: 1012.3884] [INSPIRE].

[54] M. Beccaria and A.A. Tseytlin, Partition function of free conformal fields in 3-plet representation, JHEP 05 (2017) 053 [arXiv: 1703.04460] [INSPIRE].

[55] K. Bulycheva, I.R. Klebanov, A. Milekhin and G. Tarnopolsky, Spectra of Operators in Large-N Tensor Models, Phys. Rev. D 97 (2018) 026016 [arXiv:1707.09347] [INSPIRE].

[56] M. Klemm, Tensorprodukte von Charakteren der symmetrischen Gruppe, Arch. Math. 28 (1977) 455.

[57] Y. Dvir, On the Kronecker product of Sn characters, J. Algebra 154 (1993) 125

[58] M. Clausen and H. Meier, Extreme irreduzible Konstituenten in Tensordarstellungen symmetrischer Gruppen, Bayreuth. Math. Schr. 45 (1993) 1. 